\title{
On Control Structure Design for a Walking Beam Furnace * $\dagger$
}

\author{
H. Jafari ${ }^{1}$, M. Castaño ${ }^{1}$, T. Gustafsson ${ }^{1}$ and G. Nikolakopoulos ${ }^{1,2}$
}

\begin{abstract}
The aim of this article is to introduce a novel sparse controller design for the temperature control of an experimental walking beam furnace in steel industry. Adequate tracking of temperature references is essential for the quality of the heated slabs. However, the design of the temperature control is hindered by the multivariable (non-square) dynamic behavior of the furnace. These dynamics include significant loop interactions and time delays. Furthermore, a novel datadriven model, based on real life experimental data that relies on a subspace state representation in a closed loop approach is introduced. In the sequel, the derived model is utilized to investigate the controller's structure. By applying the relative gain array approach a decentralized feedback controller is designed. However, in spite of the optimal and sparse design of the controller, there exists interaction between loops. By analyzing the interaction between the inputs-outputs with the $\Sigma_{2}$ Gramian-based interaction methodology, a decoupled multivariable controller is implied. The simulation result, based on the experimental modeling of the furnace, shows that the controller can successfully decrease the interaction between the loops and track the reference temperature set-points.
\end{abstract}

\section{INTRODUCTION}

Walking Beam Furnaces (WBFs) are broadly utilized in the modern steel industry to reheat the steel slabs to prescribed temperatures in order to change its mechanical properties. In a WBF, the slabs are walked in a cyclic movement, through heating zones of different temperatures. From an energy efficiency perspective, this process plant is one of the most energy consuming systems in the steel industry [1]. Therefore, an efficient controller is needed to minimize the energy cost for the generated heat. Additionally, in the WBF, the temperature has an important effect on the energy expenditure and the quality of the heated slabs and thus the corresponding regulation of the temperature, for each specific zone of the furnace, is desired.

In order to control the temperature in an optimal approach, initially a reliable model of the furnace is needed and this is the aim of the first contribution of this article. In the previous literature, a variety of corresponding models have been investigated. More specifically, in [2] Computational Fluid Dynamics (CFD) techniques were used for modeling, however the presented approach is computationally expensive for large industrial cases and it has more suitable results

*The work has received funding from the European Unions Horizon 2020 Research and Innovation Programme under the Grant Agreement No.636834, DISIRE

$\dagger$ Authors' preprint of paper with the same title accepted for publication in the IEEE Mediterranean Conference on Control and Automation.

${ }^{1}$ H. Jafari, M. Castaño, T. Gustafsson and G. Nikolakopoulos are with Control Engineering group, Devision of Signals and Systems, Department of Computer Science, Electrical and Space Engineering, LuleåUniversity of Technology, Sweden. geonikeltu.se at steady state, while missing to represent the transient behavior of the process.

From another approach, a considerable research in mathematical modeling, based on the physical properties, has been performed in the last decades. A simplified model, by considering first principles, was obtained in [3]. However, this model does not consider the radiative heat transfer and complex phenomena, such as turbulences. A model with less computationally complexity was introduced in [4], which is only valid for steady state operation. A novel mathematical model, based on the zone method of radiation analysis and combing this model with CFD has been performed in [5], while it was extended to a 3D approach in [6]. Although several studies have been performed for the physical modeling, little attention has been paid for the black box modeling. Since unknown disturbances effect the WBF distinctly, black box modeling may be a better approach for this industrial plant, as in the works presented in [7] and [8] where the Auto-Regressive with an eXogenous term (ARX), modeling approach for the reheating furnaces, have been adopted. However, studies on feedback loop data modeling are still lacking.

In general, the concept of Process Control is always a challenging problem, since the development of the industrial plants lead to an increasing complexity of the utilized control configurations. In the specific case of the WBF, distinct approaches to design the temperature control scheme have been introduced. Until now, the most common strategy was to design diagonal PID controllers, as the work in [9] and [10]. However, the performance of these controllers are not always satisfactory, due to the existence of large time varying delays and the high interaction between the control loops [11]. Alternative popular control schemes, relies on the framework of the Model Predictive based Control (MPC) as in [12] and [13]. These approaches, in spite of a good MPC's performance in controlling the temperature and handling the constraints, may not be practical in case of model mismatches and high plant disturbances, while for a satisfactory operation, a high number of utilized tunning parameters is needed. However, most of the previous studies do not take into account the coupling effects and the interactions of inputs-outputs for the process plant to design the proper optimal control scheme.

The main novelty of this article, stems from the utilization of structural decisions for the design of the WBF model, while additionally a novel sparse control scheme for the temperature control of the walking beam furnace is introduced. First, a novel feedback based data-driven model, by considering disturbances to the furnace, is presented based 
on real life experimental data. The obtained model is considered as a multi-variable system with a non-square transfer function. An additional novelty of this article is based on the simplification of the previous derived plant model and the corresponding study of the input-output interactions of the system by the utilization of the Relative Gain Array (RGA) theory and the Gramian based interaction measurement, $\Sigma_{2}$. Finally, the reduced obtained model is used to design the controller configuration. The designed controller is tested with real measurements of the furnace and the results were compared with the decentralized PID controller.

The rest of this article is structured as it follows. The modeling of the furnace is provided in Section II, while the input-output selection for reducing the model's order is described in the Section III. In the sequel, the controller design, based on the obtained configuration is established in the same Section, while simulation studies that prove the efficacy of the suggested scheme are depicted in Section IV. Finally, the conclusions are drawn in Section $\mathrm{V}$ that summarizes the main outcome of this article, while defining the future work directions.

\section{WBF PLANT DESCRIPTION AND MODELING}

Figure 1 depicts the schematic diagram of the experimental WBF at Swerea MEFOS AB, Luleå, Sweden. This WBF, consists of three main temperature control zones. In the WBF operation, initially the slabs enter the furnace through the hatch loading door and proceed to the first zone where are preheated. The second zone heats the slabs to the maximum desired temperature. Then, the heated slabs are entered the soaking zone (Zone 3), where the temperature of the furnace slightly increases and the temperature inside the slabs is normally distributed. Finally the slabs are discharged through the unloading door.

Each control zone includes a pair of burners that fire the light oil and are utilized as the corresponding actuators of the process. The supply rate of the oil and the air flow for the combustion process are fed to the burners at each control zone. The temperature of each zone is measured by two thermocouples, one at the wall of the furnace and the other one at the ceiling. Additionally, the oxygen $\left(\mathrm{O}_{2}\right)$ percentage inside the furnace and the internal pressure $P$ of the furnace are measured. The hot combustion gases are conveyed by two exhaust pipes located at zone 3 . These exhaust pipes recirculate the exhaust gases through the interior of the three zones in order to reuse the heat and later are fed to a heat exchanger in order to preheat the combustion air. This recirculation of the combustion gases adds complexity to the dynamics of the process for representing a physical feedback loop.

A mathematical model for the temperature of the furnace by using heat and mass balance equations was driven by [3], while for clarity and consistency of this article it will be also presented as it follows:

$$
\frac{d T_{1}}{d t}=\frac{\alpha}{\bar{c}_{1} N_{1}} F_{O 1}+\frac{\beta}{\bar{c}_{1} N_{1}} F_{A 1}\left(T_{1}-T_{\text {ref } 1}\right)
$$

$$
\begin{aligned}
& +\gamma_{1} \frac{\bar{c}_{2} P_{2}}{N_{1} \bar{c}_{1} T_{2}} \sqrt{P_{2}^{2}-P_{1}^{2}}\left(T_{1}-T_{\text {ref } 1}\right) \\
& -\gamma_{1} \frac{P_{2}}{N_{1} T_{2}} \sqrt{P_{2}^{2}-P_{1}^{2}}\left(T_{1}-T_{\text {ref } 1}\right) \\
& -\frac{1}{N_{1}}\left(T_{1}-T_{r e f}\right)\left(F_{A 1}+0.76 F_{O 1}\right) \\
& -\frac{1}{\bar{c}_{1} N_{1}} Q_{1}-\frac{1}{\bar{c}_{1} N_{1}} W_{1} \\
& \frac{d T_{2}}{d t}=\frac{\alpha}{\bar{c}_{2} N_{2}} F_{O 2}+\frac{\beta}{\bar{c}_{2} N_{2}} F_{A 2}\left(T_{2}-T_{\text {ref } 2}\right) \\
& +\gamma_{2} \frac{\bar{c}_{3} P_{3}}{N_{2} \bar{c}_{2} T_{3}} \sqrt{P_{3}^{2}-P_{2}^{2}}\left(T_{2}-T_{\text {ref } 2}\right) \\
& -\gamma_{2} \frac{P_{3}}{N_{2} T_{3}} \sqrt{P_{2}^{2}-P_{2}^{2}}\left(T_{2}-T_{\text {ref } 2}\right) \\
& -\frac{1}{N_{2}}\left(T_{2}-T_{\text {ref } 2}\right)\left(F_{A 2}+0.76 F_{O 2}\right) \\
& -\frac{1}{\bar{c}_{2} N_{2}} Q_{2}-\frac{1}{\bar{c}_{2} N_{2}} W_{2} \\
& \frac{d T_{3}}{d t}=\frac{\alpha}{\bar{c}_{3} N_{3}} F_{O 3}+\frac{\beta}{\bar{c}_{3} N_{3}} F_{A 3}\left(T_{3}-T_{\text {ref } 3}\right) \\
& -\frac{1}{N_{3}}\left(T_{3}-T_{\text {ref } 3}\right)\left(F_{A 3}+0.76 F_{O 3}\right) \\
& -\frac{1}{\bar{c}_{3} N_{3}} Q_{3}-\frac{1}{\bar{c}_{3} N_{3}} W_{3}
\end{aligned}
$$

where $T_{i} i \in 1,2,3$ is the temperature of each zone to be controlled and $T_{\text {ref }}$ is the related reference temperature. $F_{O}$, $F_{A}$ and $P$ are the oil feed flow, air feed flow and pressure correspondingly inside of each heating zone respectively. The amount of energy, which is transfered to the slabs is represented by the variables $Q$ and $W$ that imply the transfered energy to the wall of each zone of the furnace. $N$ denotes the number of moles of the furnace gas and $\bar{c}$ represents the heat capacity at each zone of the furnace. The other parameters $(\alpha, \beta, \gamma)$ are the parameters of the system to be identified. The above equations present a simplified analysis of the modeling of the furnace, without considering the turbulence effect, heat transfer by radiation and the effect of spatial domain to the temperature of each zone of the furnace. However, it can be used as conceptual illustration of the structure of the system and determined the effective inputs and the outputs of the furnace. This investigation will be later used in the data-driven modeling.

\section{A. System identification}

Subspace state-pace system identification method, is a powerful methodology to predict the dynamic of the system from measured data [14]. By assuming that the temperature at each zone of the furnace can be approximated by a linear function, the dynamics of the system can be described as:

$$
\begin{aligned}
x_{k+1} & =A x_{k}+B u_{k}+K e_{k} \\
y_{k} & =C x_{k}+D u_{k}+e_{k}
\end{aligned}
$$

where $k \geq 0$ is discrete time, $x_{k} \in \mathbb{R}^{n}$ is the state vector, $u_{k} \in \mathbb{R}^{r}$ is the input vector, $e_{k} \in \mathbb{R}^{l}$ is the zero mean 


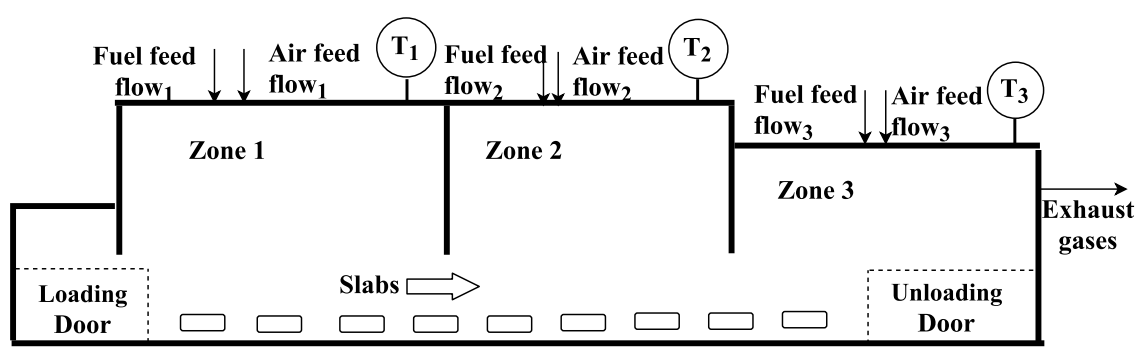

Fig. 1: The schematic block diagram of the Walking Beam Furnace under study.

white innovation process and $y_{k} \in \mathbb{R}^{m}$ represents the output vector, while $A, B, C, D$ and $K$ are constant matrices with proper dimensions. This form of representing the system for closed loop identification is a case of the so-called direct method. The direct method is preferred when operating data from the plant are available without knowledge of the controllers. The direct method ignores the closed loop in the formulation of the system, but requires that the noise models $(K)$ are sufficiently complex to represent the influence of the controller [15].

Subspace system identification refers to the concept of identifying those constant matrices directly from measured input-output data. In this article, the Numerical algorithms for the Subspace State Space System IDentification (N4SID) with a Canonical Variable Algorithm (CVA) [16] are applied to the WBF's obtained experimental data. Initially, the vector of stacked inputs, outputs and error are introduced in a future horizon as:

$$
\begin{aligned}
y_{k}^{f} & =\left[y_{k}^{T}, y_{k+1}^{T}, \ldots, y_{k+f-1}^{T}\right]^{T} \\
u_{k}^{f} & =\left[u_{k}^{T}, u_{k+1}^{T}, \ldots, u_{k+f-1}^{T}\right]^{T} \\
e_{k}^{f} & =\left[e_{k}^{T}, e_{k+1}^{T}, \ldots, e_{k+f-1}^{T}\right]^{T}
\end{aligned}
$$

where $f \in \mathbb{Z}^{+}$is arbitrary chosen by the user and determines the number of the steps ahead prediction. By the subspace definition in $(4 a, 4 b)$ the output variable can be redefined as:

$$
\begin{aligned}
& y_{k}^{f}=\Gamma x_{k}+\Phi u_{k}^{f}+\Psi e_{k}^{f} \\
& \text { where } \Gamma=\left[\begin{array}{c}
C \\
C A \\
\vdots \\
C A^{f-1}
\end{array}\right] \text { is the observability matrix and } \\
& \begin{aligned}
\Phi= & {\left[\begin{array}{ccccc}
D & 0 & \cdots & & 0 \\
C B & D & & & \\
C A B & C B & \ddots & & \vdots \\
\vdots & & \ddots & & 0 \\
C A^{f-2} B & & & C B & D
\end{array}\right], } \\
\Psi & =\left[\begin{array}{ccccc}
I & 0 & \cdots & & 0 \\
C K & I & & & \\
C A K & C K & \ddots & & \vdots \\
\vdots & & \ddots & & 0 \\
C A^{f-2} K & & & C K & I
\end{array}\right]
\end{aligned}
\end{aligned}
$$

The first step in this method is to estimate the state vectors by the vectors of measured inputs and outputs. Thus, the states matrix can be assumed as a function of $j$-steps-back inputs and outputs data as:

$$
\hat{x}_{k}=\Upsilon p_{k}
$$

where $\Upsilon$ is a matrix with unknown coefficient to be estimated and

$$
p_{k}=\left[\begin{array}{cccc}
y_{k-1}^{T} & y_{k-2}^{T} & \cdots & y_{k-j}^{T} \\
u_{k-1}^{T} & u_{k-2}^{T} & \ldots & u_{k-j}^{T}
\end{array}\right]^{T}
$$

This estimation can be done by regressing $y_{k}^{f}$ on $p_{k}$ and $u_{k}^{f}$ from equation (6) and obtaining $\hat{\Gamma \mathcal{K}}$ and $\hat{\Phi}$.

The next step is to estimate $\Upsilon$ and as a result the states vector $\hat{x}_{k}$. In order to do that, first the effect of the future input on the future output should be removed by the linear regression problem defined as:

$$
z_{k} \triangleq y_{k}^{f}-\hat{\Phi} u_{k}^{f} \approx \Gamma \Upsilon p_{k}+\Psi e_{k}^{f}
$$

Then $\Upsilon$ can be estimated by solving the singular value decomposition on the correlation analysis on $z_{k}$ and $p_{k}$ as it follows:

$$
U S V^{T}=\left(\hat{R}_{z z}\right)^{-\frac{1}{2}}\left(\hat{R}_{z p}\right)\left(\hat{R}_{p p}\right)^{-\frac{1}{2}}
$$

where $\hat{R}_{z p}=\frac{1}{N} \sum_{i=1}^{N} z_{k} p_{k}^{T}$ is the correlation between $z_{k}$ and $p_{k}$, with $U$ and $\bar{V}$ to be the orthonormal matrices of left and right singular vectors and $S$ is the diagonal matrices of singular values. Solving the above equation leads to estimating the CVA estimate of $\Upsilon$. Finally, after estimating the states matrices by equation (7), the parameters of the system, defined by the matrices $A, B, C, D$ and $K$ can be estimated by the linear regression of state-space model in (4a and 4b) (see [16], [17]).

Unlike classical prediction methods, such as the ARX that optimize effectively for one-step-ahead prediction, the presented algorithm can optimize a $k$-steps-ahead prediction [18]. This property will reduce a bias in the prediction and will conclude in a more accurate model of the system, which will be later used by the control scheme.

\section{B. Model validation and simulation results}

In order to identify the dynamics of the system by a data-driven methodology, a set of data from an online campaign for several days of the WBF's operation 
at MEFOS were utilized. After data cu-ration, considering equations (1, 2 and 3 ) and the available measurements the variables of the inputs and outputs of the furnace are selected. Since only the pressure of the whole furnace is measured by a sensor, the effect of this input is neglected in the identification process. Moreover, the exhaust flow of gases is consider as an another input applied to the furnace. Therefore, the inputs for the system identification are $u=\left[F_{O 1}, F_{A 1}, F_{O 2}, F_{A 2}, F_{O 3}, F_{A 3}, F_{\text {exhaust }}\right]^{T}$ and the corresponding outputs are $y=\left[T_{1}, T_{2}, T_{3}\right]^{T}$.

For avoiding an over-fitting of the data prediction, two sets of data are selected: the training and testing. The training sets are chosen in a way that capture all the important operating condition and consider a general case where both hatch doors are open. For simplicity, it is assumed that there is no feed through and this results for the matrix $D=0$. The initial estimated order of the system is determined by Akaike Information Criterion (AIC). Figure 2 depicts the 20 steps ahead prediction of temperature of each zone of the WBF on the validation data set, with a sampling time of 10 seconds.

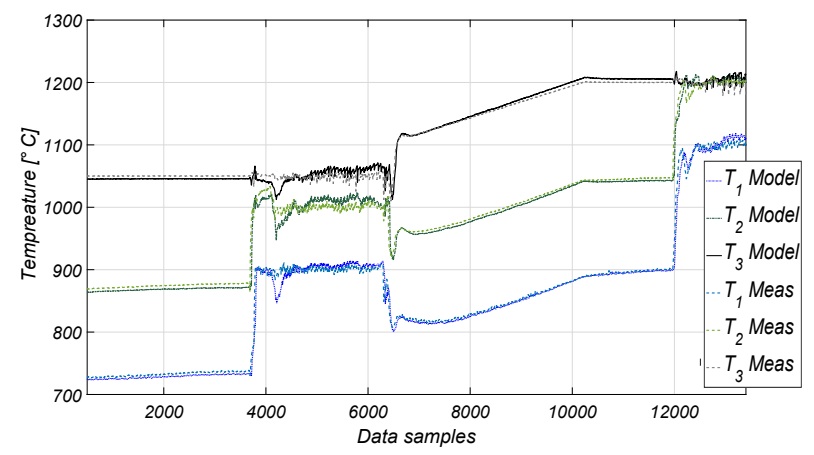

Fig. 2: Validation of model over testing data set with a 20 steps ahead prediction. The dashed red line is the measurement data, while the solid blue line represents the model.

From the obtained results, it is straight forward that the resulted model has a very good performance in representing the dynamics of the WBF, while at the same time has a very accurate matching. Furthermore, Figure 3 presents the change of Predicted Root Mean Square Error (PRMSE) of each zone temperature for different $k$-steps-ahead prediction.

Obviously, by increasing the prediction horizon it results in a bigger prediction error and a less accuracy of the model. However, since the operating time for this furnace is in hours, this model can sufficiently predict the dynamic of the WBF.

\section{CONTROL CONFIGURATION SELECTION}

The design of a control system for a multivariable process usually involves the design of a control configuration prior to the synthesis of controller parameters [20]. In Control Configuration Selection (CCS) one approach is to simplify the complete system model by selecting the most important input-output interconnections of the system and reduced the complexity of the model from the selected components [21].

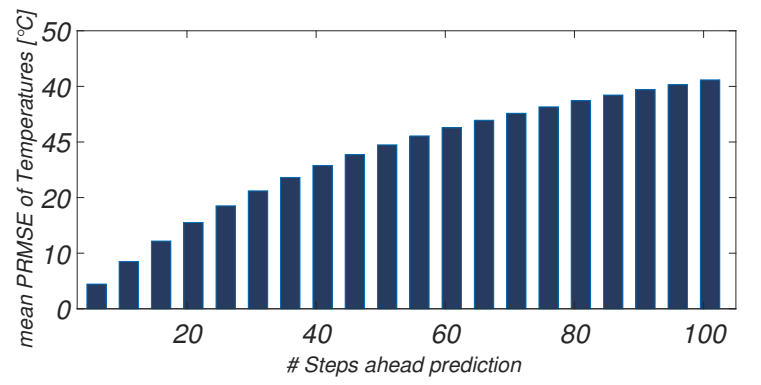

Fig. 3: Illustration of ability of the prediction by changing the prediction horizon. The mean of PRMSE for output temperatures is compared with changing the prediction horizon.

The derived simplified model can be later used to design the controller. Moreover, CCS can be utilized to design a sparse and less complex controller that considers the interconnections between all the loops.

In most of the industrial applications such as the WBF, the control configuration is designed by using previous knowledge of the system, rules of thumb or even geographical proximity between sensors and actuators [22]. Here, by applying CCS methods such as classical Relative Gain Array (RGA) and the $\Sigma_{2}$ Gramian-based interaction measure on the obtained model of the WBF, a systematic approach to design a sparse control configuration is desired.

\section{A. Quantifying controllability and observability with grami- ans}

The controllability $(P)$ and observability $(Q)$ gramians for the system in (4a) and (4b) can be calculated by solving the following Lyapunov equations [23]:

$$
\begin{aligned}
& A P+P A^{T}+B B^{T}=0 \\
& A^{T} Q+Q A+C^{T} C=0
\end{aligned}
$$

It is well-understood that the gramians can be used to quantify the system controllability and observability due to their energy interpretations. The eigenvalues of $P$ or $Q$ are often used for this quantification, since the states that are difficult to reach are in the span of eigenvectors of $P$, which correspond to small eigenvalues, and the states which are difficult to observe are in the span of eigenvectors of $Q$ which correspond to small eigenvalues [24]. An issue is that the gramians depend on the realization of the process (selection of states).

In the sequel of this section, gramians will be used to perform model reduction, input selection and control configuration selection.

\section{B. Model order reduction}

Since numerical errors have been observed in the calculations of the gramians, a balanced truncation has been performed in order to reduce the number of the states of the model [25].

The states that are related to the number of Hankel Singular values $10^{-4}$ times smaller than the largest singular value are removed, since an upper bound on the approximation 
error can be calculated from the sum of the disregarded Hankel Singular values [26].

\section{Input Selection}

The system presents 3 temperature measurements and 3 actuators grouped in 3 oil flows, 3 air flows and exhaust flow. It is of convenience to perform input selection in order to square down the plant for applying control techniques for squared systems. The controllability gramian can be used for the selection of $p$ inputs by evaluating the controllability of the reduced systems resulting from choosing any $p$ combination of inputs chosen from the $r$ total number of inputs [27]. If the maximum eigenvalue of the controllability gramian is considered, then the reduced $B$ matrix $\left(B_{p}\right)$, resulting from the optimal selection of $p$ actuators is obtained from the following integer optimization:

$$
B_{p}=\arg . \max _{B i=B(i,:), i \in\left(\begin{array}{l}
r \\
p
\end{array}\right)} \bar{\lambda}\left(P\left(A, B_{i}, C\right)\right)
$$

where $\bar{\lambda}\left(P\left(A, B_{i}, C\right)\right)$ is the maximum eigenvalue $(\bar{\lambda})$ of the controllability matrix $P\left(A, B_{i}, C\right)$ of the minimal realization of the reduced system formed by picking the columns of $B$ indicated by the vector $i$. The vector $i$ belongs to the set $\left(\begin{array}{l}r \\ p\end{array}\right)$ of $p$-combinations of the first $r$ integers.

\section{Controller structure and controller design}

After the selection of the reduced and simplified model, CCS can be performed to the identified interaction between the loops, while RGA which was introduced by Bristol in [28] can be used for initial analysis of the system. By definition, the RGA of a continuous process $G(s)$ with equal number of inputs and outputs is defined as:

$$
\Lambda=G(0) \otimes G(0)^{-T}
$$

where $G(0)^{-T}$ is the transpose of the inverse of $G(0)$ and $\otimes$ indicates element by element multiplication. Based on the RGA pairing rules, it is preferred to select the pairing with the related RGA value close to 1 . Since, this can be interpreted that there is no interaction effects in that pairing. Besides, pairings with negative values of the RGA should be avoided, since it means that the gain of the subsystem changes it sign when all the other loops are closed.

However, the RGA is only applicable in the designed of decentralized control configurations, where sensors and actuators are grouped in pairs and the loops are closed using only Single-Input-Single-Output controllers. This limitation leads to improve the decision process for measurement/actuator pairing with the gramian-based interaction measurements such as $\Sigma_{2}$ [29].

By assuming the state space representation in (4a) and (4b). The $\Sigma_{2}$ can be defined as:

$$
\Sigma_{2}=\frac{\left\|G_{i j}\right\|_{2}}{\Sigma_{h, l=1}^{m, r}\left\|G_{h l}\right\|_{2}}
$$

where $\left\|G_{i j}\right\|_{2}$ is the $\mathcal{H}_{2}$ norm of the system transfer function $G_{i j}$. Since $\Sigma_{2}$ is sensitive to input-output scaling, for accuracy of the calculation it is required to scale the model as [23]:

$$
G(s)=D_{e}^{-1} \hat{G}(s) D_{u}
$$

where $D_{e}$ is the largest allowed control error and $D_{u}$ is the largest allowed input change and $\hat{G}$ indicates the unscaled transfer function.

The I/O pairing rules using this method is generally to select the elements of $\Sigma_{2 m \times m}$ that have the value greater than the average $\frac{1}{m^{2}}$. However, this would be a preliminary configuration, which has to be tested, leading to a possible redesign in favor to a configuration with larger contribution [30].

Additionally, $\Sigma_{2}$ gives a measure of output controllability, since the $\mathcal{H}_{2}$-norm can be calculated from the gramians as:

$$
\|G(s)\|_{2}=\sqrt{\operatorname{trace}\left(C P C^{T}\right)}=\sqrt{\operatorname{trace}\left(B^{T} Q B\right)}
$$

\section{Simulation Results}

The input selection for the WBF is performed by evaluating all the possible subsystems with $p=3$ actuators and by choosing the subsystem that leads to the larger value of controllability $(\bar{\lambda}(P))$. After this analysis, the subsystem formed by the 3 oil actuators: $\left\{F_{O 1}, F_{O 2}, F_{O 3}\right\}$ and the 3 temperature measurements has been found to be the combination, which achieves the largest maximum eigenvalue of the controllability gramian. The control action on the air flows will be determined as a ratio from the control action of the oil flows following the stoichiometric relationship given by the combustion reaction.

The reduced subsystem model indicates that the most influential inputs to control the WBF are the fuel feed flow at each zone of the furnace and the effect of air feed flows and the exhaust of gases can be neglected to design the sparse controller. This statement can also be confirmed by observing the data sets of the fuel feed flow and the air feed flow. From Figure 4 it can be seen that from Pearson's linear correlation coefficients [15] there is a high correlation between the Fuel feed flows and air feed flows (coefficient of correlation $\geq 0.5$ ).

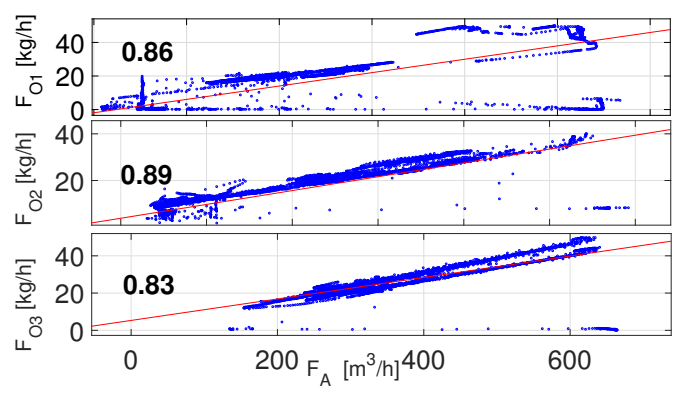

Fig. 4: Correlation between the fuel feed flow and air feed flow for each zone of the furnace. The value of Pearson correlation coefficient is shown on each sub-figure. 
The RGA is utilized to design the decentralized control configuration initially. The RGA for the subsystem model can be derived as:

$$
\lambda=\left[\begin{array}{ccc}
F_{O 1} & F_{O 2} & \multicolumn{1}{c}{F_{O 3}} \\
\hline 1.86 & -1.77 & 0.91 \\
0.07 & 4.34 & -3.41 \\
-0.93 & -1.57 & 3.5
\end{array}\right] \quad \begin{aligned}
& T_{1} \\
& T_{2} \\
& T_{3}
\end{aligned}
$$

Based on above calculation and the pairing rules mentioned in section III, the most appropriate pairing for design of the decentralized controller are: $F_{O 1}-T_{1}, F_{O 2}-T_{2}, F_{O 3}-T_{3}$.

However, this controller involved the selection of values of RGA larger than 1, which are related to an ill-conditioned system. Also, RGA is unable to capture other loops perturbations. Thus, it can be deduced that the WBF under study in this work, will be hard to control with decentralized controller.

In order to analyze the interaction between all the loops, $\Sigma_{2}$ can be calculated from the scaled model as:

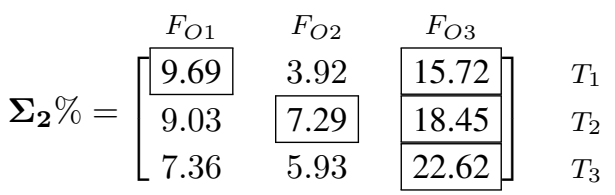

With the average contribution of $11.11 \%(1 / 9)$, the selected elements to design multi-variable controller are: $\left\{F_{O 1}, F_{O 3}-\right.$ $\left.T_{1}\right\},\left\{F_{O 2}, F_{O 3}-T_{2}\right\}$ and $\left\{F_{O 3}-T_{3}\right\}$. This investigation results controller matrix $C=\left(\begin{array}{ccc}c_{11} & 0 & c_{13} \\ 0 & c_{22} & c_{23} \\ 0 & 0 & c_{33}\end{array}\right)$. The elements $c_{13}$ and $c_{23}$ can be designed by feed forward actions. Figure 5 presents the comparison between the step response of the designed decentralized controller and the multi-variable controller for the WBF in the feedback loop. It can be seen that the decoupled multi-variable system can sufficiently decreased the interaction between the other loops. The structure of the feed back controller is illustrated in Figure 6.

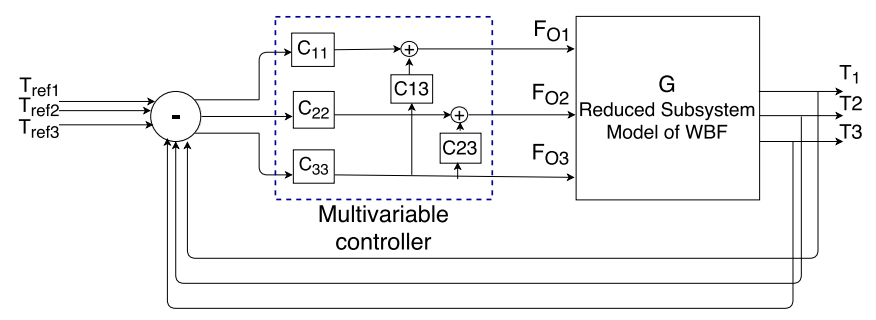

Fig. 6: The schematic of the designed multi-variable controller for WBF in the feedback loop.

To validate the designed multi-variable controller, a simulation on the experimental data of WBF was performed. As shown in Figure 7, after the change in the set points the controller is able to stabilized around the set-points after $120[\mathrm{~min}]$.

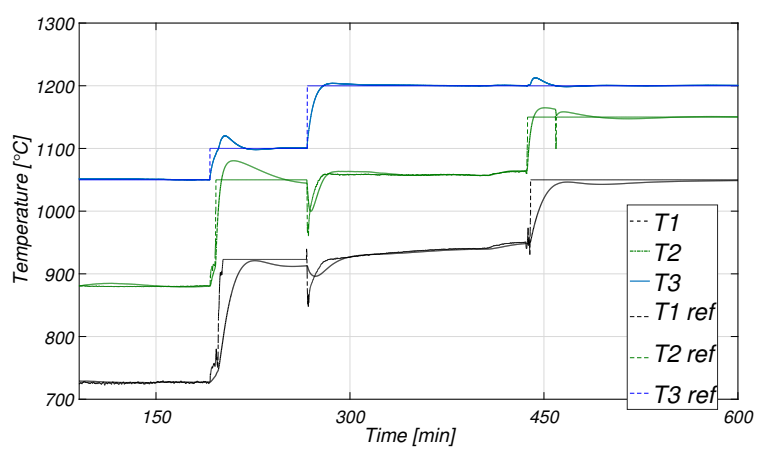

Fig. 7: Validation of the designed controller in the feedback loop on the experimental set points. The dashed lines indicate the reference temperature of each zones

\section{CONCLUSIONS}

The application of control structural design on the steel industrial plant, walking beam furnace is demonstrated in this article. Due to the high interaction between multiple inputsoutputs of this multi-variable plant, temperature control of this reheating furnace is a challenging task. A subspace state space data-driven modeling was implemented that can estimate the dynamic behavior of the WBF. This model has multiple control actions and a non-square transfer function. Control configuration methods, derived the most influential inputs to the outputs of the system and a subsystem of $3 \times 3$ transfer function is derived. The reduced model is initially analyzed by the RGA and the decentralized controller is designed. However, based on the produced results, the decentralized controller can not sufficiently control the temperature of each zone of the furnace. A gramian based interaction measurement $\Sigma_{2}$ recommends a sparse controller structure. The validation results of this suggested controller, successfully control the temperature of each zone of the furnace and track the references set points.

However, control design for this furnace is a trade off against the achieved performance and the cost of obtaining and maintaining the controllers, actuators and sensors. The advantage of the designed sparse controller is its ability to minimize this cost and the corresponding reduced complexity of the proposed control scheme, while achieving a sufficient performance. As a future work, this performance can be compared with a centralized multi-variable controller. Moreover, in extension of this work, the control structure design can be applied for the combustion process of this walking beam furnace.

\section{ACKNOWLEDGEMENTS}

This research was supported by the European Unions Horizon 2020 Research and Innovation Programme under the Grant Agreement No.636834, DISIRE. 


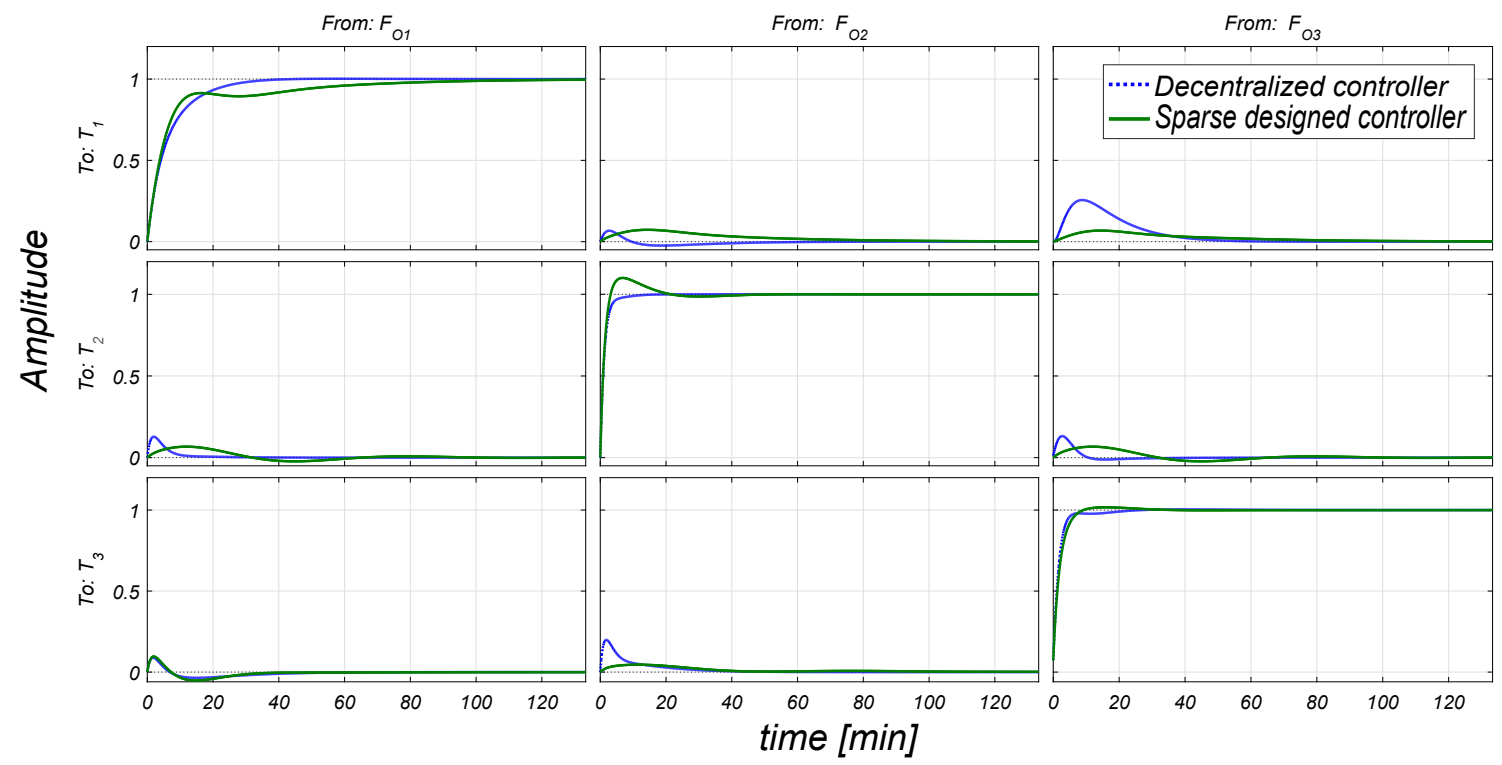

Fig. 5: The comparison between the step response of decentralized controller and the sparse multi-variable controller in the feedback loop. The decentralized controller results in perturbation between the loops.

\section{REFERENCES}

[1] Nguyen, Xuan Manh, et al. "Temperature control of reheating furnace based on distributed model predictive control." System Theory, Control and Computing (ICSTCC), 2014 18th International Conference. IEEE, 2014.

[2] Falcitelli, M., S. Pasini, and L. Tognotti. ”Modelling practical combustion systems and predicting NOx emissions with an integrated CFD based approach." Computers and chemical engineering 26.9 (2002): 1171-1183.

[3] Ko, Hyun Suk, et al. "Modeling and predictive control of a reheating furnace." American Control Conference, 2000. Proceedings of the 2000. Vol. 4. IEEE, 2000.

[4] Han, Sang Heon, Seung Wook Baek, and Man Young Kim. "Transient radiative heating characteristics of slabs in a walking beam type reheating furnace." International Journal of Heat and Mass Transfer 52.3 (2009): 1005-1011.

[5] $\mathrm{Hu}$, Yukun, et al. "Zone modelling coupled with dynamic flow pattern for the prediction of transient performance of metal reheating." Proceeding of AISTech2014the iron and steel technology conference and exposition, Indianapolis, USA, May. 2014

[6] $\mathrm{Hu}$, Yukun, et al. "Development of a first-principles hybrid model for large-scale reheating furnaces." Applied Energy 173 (2016): 555-566.

[7] $\mathrm{Xu}$, Zhe, et al. "Research on Temperature Optimal Control for the Continuous Casting Billet in Induction Heating Process Based on ARX Model." Proceedings of 2013 Chinese Intelligent Automation Conference. Springer Berlin Heidelberg, 2013.

[8] Van Ditzhuijzen, Gustaaf, Dirk Staalman, and Arnold Koorn. "Identification and model predictive control of a slab reheating furnace." Control Applications, 2002. Proceedings of the 2002 International Conference on. Vol. 1. IEEE, 2002.

[9] Leden, Bo. "A control system for fuel optimization of reheating furnaces." Scand. J. Metall. 15.1 (1986): 16-24.

[10] Srisertpol, Jiraphon, et al. "Estimation of the mathematical model of the reheating furnace walking hearth type in heating curve up process." International Journal of mathematical models and methods in applied sciences 1 (2011): 167-174.

[11] Feliu-Batlle, Vicente, Raul Rivas-Perez, and Fernando J. CastilloGarca. "Simple fractional order controller combined with a Smith predictor for temperature control in a steel slab reheating furnace." International Journal of Control, Automation and Systems 11.3 (2013): 533-544.

[12] Nguyen, Xuan Manh, et al. "Temperature control of reheating furnace based on distributed model predictive control." System Theory, Control and Computing (ICSTCC), 2014 18th International Conference. IEEE, 2014.

[13] Nederkoorn, Eelco, P. Wilgen, and Jan Schuurmans. "Nonlinear model predictive control of walking beam furnaces." Proceedings of the 1st International Conference on Energy Efficiency and CO. Vol. 2. 2011

[14] Ljung, Lennart. System identification. John Wiley \& Sons, Inc., 1999.

[15] Kendall, Maurice George. "The advanced theory of statistics." The advanced theory of statistics. 2nd Ed (1946).

[16] Van Overschee, Peter, and Bart De Moor. "N4SID: Subspace algorithms for the identification of combined deterministic-stochastic systems." Automatica 30.1 (1994): 75-93.

[17] Jansson, Magnus. "Subspace identification and ARX modeling." Proceedings of SYSID 2003 (2003).

[18] Viberg, Mats. "Subspace-based methods for the identification of linear time-invariant systems." Automatica 31.12 (1995): 1835-1851.

[19] Urban Forssell, Lennart Ljung, Closed-loop identification revisited, Automatica, Volume 35, Issue 7, 1999, Pages 1215-1241.

[20] Morari, Manfred, Yaman Arkun, and George Stephanopoulos. "Studies in the synthesis of control structures for chemical processes: Part I: Formulation of the problem. Process decomposition and the classification of the control tasks. Analysis of the optimizing control structures." AIChE Journal 26.2 (1980): 220-232.

[21] Van De Wal, Marc, and Bram De Jager. "A review of methods for input/output selection." Automatica 37.4 (2001): 487-510.

[22] M. van de Wal and B. de Jager, "Control structure design: a survey," American Control Conference, Proceedings of the 1995, Seattle, WA, 1995, pp. 225-229 vol.1.

[23] Skogestad, S., and Postlethwaite, I. (2007). Multivariable feedback control: analysis and design. New York: Wiley.

[24] Antoulas, A. C. (2001). Frequency domain representation and singular value decomposition.EOLSS Contribution 6.43.13.4.

[25] Safonov, M. G., and Chiang, R. Y. (1988, June). A Schur method for balanced model reduction. In IEEE American Control Conference, 1988 (pp. 1036-1040).

[26] Glover, K. All optimal Hankel-norm approximations of linear multivariable systems and their $\mathrm{L}, \infty$-error bounds, (1984) International journal of control, 39(6), 1115-1193.

[27] Georges, D. (1995). The use of observability and controllability gramians or functions for optimal sensor and actuator location in finitedimensional systems. In Decision and Control, Proceedings of the 34th IEEE Conference on (Vol. 4, pp. 3319-3324).

[28] Bristol, Edgar. "On a new measure of interaction for multivariable process control." IEEE transactions on automatic control 11.1 (1966): 133-134. 
[29] Birk, Wolfgang, and Alexander Medvedev. "A note on gramian-based interaction measures." European Control Conference (ECC), 2003. IEEE, 2003.

[30] Salgado, Mario E., and Arthur Conley. "MIMO interaction measure and controller structure selection." International Journal of Control 77.4 (2004): 367-383. 